\title{
Isoscalar compression modes in relativistic random phase approximation
}

\author{
Zhong-yu Ma ${ }^{1 *}$, Nguyen Van Giai ${ }^{2}$, A. Wandelt ${ }^{3}$, D. Vretenar ${ }^{3 \dagger}$ and P. Ring ${ }^{3}$ \\ 1. China Institute of Atomic Energy, Beijing, P.R. of China \\ 2. Institut de Physique Nucléaire, IN2P3-CNRS, F-91406 Orsay Cedex, France \\ 3. Physics Department, Technical University Munich, D-85748 Garching, Germany
}

(22 March 2000)

\begin{abstract}
A fully consistent relativistic RPA calculation is performed for the monopole and dipole compression modes in nuclei. The emphasis is put on the effects of Dirac sea states which are generally neglected in relativistic RPA calculations. It is found that these effects can be quite important for the isoscalar monopole mode. The main contributions from the pairs of Fermi to Dirac sea states are through the exchange of the scalar meson, while the vector mesons play a negligible role. Numerical results of relativistic RPA are checked with the constrained relativistic mean field model in the monopole case. A good agreement beteween monopole energies calculated in RRPA and in time-dependent relativistic mean field approach is achieved. For the monopole compression mode, a comparison of experimental and calculated energies gives a value of $250 \sim 270 \mathrm{MeV}$ for the nuclear matter incompressibility. A large discrepancy remains between theory and experiment in the case of the dipole compression mode.
\end{abstract}

Keywords: Relativistic random phase approximation; Relativistic meanfield; Giant resonances; Compression modulus

*also Institute of Theoretical Physics, Beijing, P.R. of China

${ }^{\dagger}$ On leave from University of Zagreb, Croatia 


\section{INTRODUCTION}

The linear response of a system to a weak external field can be calculated in the random phase approximation (RPA) which is one of the main theoretical approaches to study the nuclear giant resonances in a microscopic way [1,2]. In the non-relativistic approach the equivalence between the RPA and the small amplitude limit of the time-dependent Hartree-Fock approximation is well-known. Numerous RPA calculations based on HartreeFock single-particle spectra have been performed using effective nucleon-nucleon interactions such as Skyrme and Gogny forces. These calculations are very successful in describing the collective excitations and giant resonances in various nuclei in a framework which is consistent in the sense that both mean field and residual interaction come from the same effective force.

In recent years the relativistic mean-field (RMF) theory with non-linear effective Lagrangians has received considerable attention. The RMF has met great success in describing the bulk properties of nuclei. One of the advantages of the RMF approach is that the same effective Lagrangian with only a few free parameters could predict the ground state properties of nuclei covering the whole periodic table and also exotic nuclei from the proton drip line to the neutron drip line. The success of the RMF encouraged to investigate the extensions of the RMF approach to study dynamical processes, especially the collective giant resonances. The linear response of a system to an external field can be calculated in the relativistic RPA (RRPA) which is again a small amplitude limit of the time-dependent RMF (TDRMF) theory [3].

In the relativistic approach, it is known from nuclear matter studies that self-consistency is extremely important. Self-consistent wave functions where the lower components in the Dirac spinor are enhanced by an effective mass must be adopted in the Dirac-Brueckner Hartree-Fock calculations [4]. Two questions of consistency appear in the RRPA. First, the particle-hole interaction must be determined from the same Lagrangian used in the RMF. The meson nonlinear self-interaction terms in the effective Lagrangian bring in densitydependent interactions which are essential for a quantitative description of the nuclear properties, especially the nuclear matter compression modulus. The meson propagators with nonlinear terms have been worked out in Refs. [5],6]. The second question of consistency is that the RRPA must be consistent with the RMF. It is assumed in the RMF that only positive energy states contribute to the nucleon self-energies (no-sea approximation). A complete relativistic Hartree approximation would have to include the contributions from Dirac states, which are divergent and a renormalization procedure is required [7]. Unfortunately, a proper treatment of the relativistic Hartree approach in finite nuclei is too complicated and one has to work in the no-sea approximation. Nevertheless, a consistent RRPA built on an RMF ground state must deal in principle with the Dirac states. This is because RRPA is a small amplitude limit of TDRMF and the density change $\delta \rho(t)$ at time $t$ has necessarily matrix elements not only between positive energy particle-hole pairs but also between pairs formed from Dirac sea states and occupied Fermi sea states of the static solution. Thus, an RRPA calculation consistent with the no-sea approximation should include both positive energy and negative energy single-particle states. Although it has been known before 8] that the inclusion of the Dirac states ensures current conservation and decoupling of the spurious state, it was thought that the contribution to the RRPA energies and strengths 
from the Dirac states would be negligible. Therefore, the observables of interest (excitation energies and transition densities) would remain the same with and without including the Dirac states. Most previous works in studying the response functions of nuclei included the positive energy particle-hole pairs only. With this prescription, discrepancies between the RRPA and the TDRMF appeared, especially in the isoscalar monopole modes, which could not be understood for some time [9, 10]. The main purpose of this paper is to clarify this puzzle and to examine the effects of the Dirac states on the calculation of compression modes in the RRPA approach. We analyze the origin of the contributions from Dirac states in the study of the isoscalar nuclear giant resonances. To verify that the calculation of RRPA with Dirac states is the correct and consistent procedure we compare the inverse energy-weighted sum rules calculated in RRPA and in constrained RMF calculations. A good agreement can be obtained only when Dirac states are included. Results concerning isoscalar giant monopole resonances (ISGMR) and isoscalar giant dipole resonances (ISGDR) in nuclei are presented.

The outline of this paper is as follows. The formalism of the relativistic linear response is outlined in Sec.II where we investigate the implications of including Dirac states in the RRPA equations. In Sec.III the constrained RMF method is used to calculate isoscalar monopole polarizabilities which are compared to those deduced from RRPA. Results of calculations of ISGMR and ISGDR in medium and heavy nuclei are presented in Sec.IV. Finally, Sec.V contains a summary of the main results.

\section{DIRAC STATES IN THE RRPA}

We start from the Hartree single-particle Green's function which is defined by:

$$
G_{H}(x, y)=-i\left\langle 0\left|T\left(\Psi_{H}(x) \bar{\Psi}_{H}(y)\right)\right| 0\right\rangle
$$

where $x \equiv(t, \mathbf{r}), \Psi_{H}(x)$ is the Hartree field operator and $|0\rangle$ is the uncorrelated Hartree ground state. Then, the Dyson equation for the nucleon propagator is

$$
G_{H}(x, y)=G_{0}(x, y)+\int d^{4} x_{1} d^{4} x_{2} G_{0}\left(x, x_{1}\right) \Sigma^{H}\left(x_{1}, x_{2}\right) G_{H}\left(x_{2}, y\right)
$$

where the nucleon self-energy due to meson-nucleon couplings can be calculated in the Hartree approximation including only the tadpole Feynman diagram:

$$
\begin{aligned}
\Sigma^{H}(x, y)= & \delta^{4}(x-y)\left\{-i g_{s}^{2} \int d^{4} x_{1} D_{\sigma}\left(x-x_{1}\right) \operatorname{Tr}\left[G_{H}\left(x_{1}, x_{1}^{+}\right)\right]\right. \\
& \left.-i \gamma_{\mu} g_{v}^{2} \int d^{4} x_{1} D_{\omega}^{\mu \nu}\left(x-x_{1}\right) \operatorname{Tr}\left[\gamma_{\nu} G_{H}\left(x_{1}, x_{1}^{+}\right)\right]\right\} \\
= & \delta^{4}(x-y)\left[\Sigma_{s}(x)-\gamma_{\mu} \Sigma_{v}^{\mu}(x)\right]
\end{aligned}
$$

where $x^{+}$refers to the time-ordering prescription and $D_{\sigma}, D_{\omega}$ are the meson propagators of $\sigma$ and $\omega$, respectively. For the convenience of notations, we write explicitly only the $\sigma$ and

$\omega$ mesons in this section but the actual calculations of the next sections contain also the $\rho$ meson and photon fields.

The Hartree Green's function in a standard spectral representation is expressed as: 


$$
\begin{aligned}
G_{H}\left(\mathbf{r}_{1}, \mathbf{r}_{2} ; E\right)= & \sum_{\alpha} \frac{\phi_{\alpha}\left(\mathbf{r}_{1}\right) \bar{\phi}_{\alpha}\left(\mathbf{r}_{2}\right)}{E-\varepsilon_{\alpha}+i \eta}+\sum_{\bar{\alpha}} \frac{\phi_{\bar{\alpha}}\left(\mathbf{r}_{1}\right) \bar{\phi}_{\bar{\alpha}}\left(\mathbf{r}_{2}\right)}{E-\varepsilon_{\bar{\alpha}}-i \eta} \\
& +\sum_{h<\varepsilon_{F}} \phi_{h}\left(\mathbf{r}_{1}\right) \bar{\phi}_{h}\left(\mathbf{r}_{2}\right)\left(\frac{1}{E-\varepsilon_{h}-i \eta}-\frac{1}{E-\varepsilon_{h}+i \eta}\right) \\
\equiv & G_{H}^{F}+G_{H}^{D},
\end{aligned}
$$

where we denote by $\alpha$ all positive energy states $\left(\varepsilon_{\alpha}>0\right), \bar{\alpha}$ all negative energy states $\left(\varepsilon_{\bar{\alpha}}<0\right)$ and $h$ the states in the Fermi sea. The unoccupied positive energy states will be denoted by $p$ in the following expressions. The superscripts $F$ and $D$ refer to the Feynman and density-dependent parts of the Green's function, respectively. When one calculates the nucleon self-energy of Eq.(3) with this Green's function Eq.(4), all occupied states $\bar{\alpha}$ and $h$ will contribute. The contributions from occupied Dirac states are divergent and therefore, a renormalization procedure is necessary. This corresponds to the so-called relativistic Hartree approximation. In contrast, the RMF approach which is the framework we adopt here assumes no contributions to the nucleon self-energies from the Dirac states. This no-sea approximation corresponds to a modified single-particle Green's function, where the poles $\varepsilon_{\bar{\alpha}}+i \eta$ for negative energy states are shifted to $\varepsilon_{\bar{\alpha}}-i \eta$ in Eq.(4) [8]. This gives a modified Green's function $\tilde{G}_{H}$ built on a complete set of single-particle states and it satisfies the Dyson equation Eq.(2). A consistent RRPA built on the RMF ground state has to start from this modified single-particle Green's function.

The RRPA method used in this paper is described in detail in Refs. [11.50 with just the replacement of $G_{H}$ by $\tilde{G}_{H}$. The linear response of a system to an external field is given by the imaginary part of the retarded polarization operator:

$$
R(Q, Q ; \mathbf{k}, E)=\frac{1}{\pi} \operatorname{Im} \Pi^{R}(Q, Q ; \mathbf{k}, \mathbf{k} ; E),
$$

where $\mathrm{Q}$ is a one-body operator represented by a $4 \times 4$ matrix. The polarization operator can be obtained by solving the Bethe-Salpeter equation:

$$
\begin{aligned}
& \Pi\left(P, Q ; \mathbf{k}, \mathbf{k}^{\prime}, E\right)=\Pi_{0}\left(P, Q ; \mathbf{k}, \mathbf{k}^{\prime}, E\right) \\
& -\sum_{i} g_{i}^{2} \int d^{3} k_{1} d^{3} k_{2} \Pi_{0}\left(P, \Gamma^{i} ; \mathbf{k}, \mathbf{k}_{1}, E\right) D_{i}\left(\mathbf{k}_{1}-\mathbf{k}_{2}, E\right) \Pi\left(\Gamma_{i}, Q ; \mathbf{k}_{2}, \mathbf{k}^{\prime}, E\right) .
\end{aligned}
$$

In this equation the index $i$ runs over $\sigma, \omega$ and $\rho$ mesons, $g_{i}$ and $D_{i}$ are the corresponding coupling constants and meson propagators. The meson propagators for non-linear models are non-local in momentum space and they have to be calculated numerically. The detailed expressions of the $D_{i}\left(\mathbf{k}_{1}-\mathbf{k}_{2}, E\right)$ can be found in Refs. [5, 6]. The $\Gamma_{i}$ 's are the $4 \times 4$ matrices $1, \gamma_{\mu}$ and $\gamma_{\mu} \vec{\tau}$ for $i=\sigma, \omega$ and $\rho$, respectively. Notice that the space-like parts of vector mesons play no role in the static ground states, but they provide attractive contributions to the particle-hole residual interaction. The kernel of the Bethe-Salpeter equation has a simple form in the RMF approximation:

$$
\begin{aligned}
\Pi_{0}\left(P, Q ; x_{1}, x_{2}\right) & =i\left\langle 0\left|T\left[\bar{\Psi}_{H}\left(x_{1}\right) P \Psi_{H}\left(x_{1}\right) \bar{\Psi}_{H}\left(x_{2}\right) Q \Psi_{H}\left(x_{2}\right)\right]\right| 0\right\rangle \\
& =i \operatorname{Tr}\left[P \tilde{G}_{H}\left(x_{1}, x_{2}\right) Q \tilde{G}_{H}\left(x_{2}, x_{1}\right)\right] .
\end{aligned}
$$

In the spectral representation, the unperturbed polarization operator has the following retarded form: 


$$
\Pi_{0}^{R}\left(P, Q ; \mathbf{k}, \mathbf{k}^{\prime}, E\right)=\sum_{h, a=p, \bar{\alpha}}\left[\frac{\left\langle\bar{\Psi}_{h}|P| \Psi_{a}\right\rangle\left\langle\bar{\Psi}_{a}|Q| \Psi_{h}\right\rangle}{E-\left(\varepsilon_{a}-\varepsilon_{h}\right)+i \eta}+\frac{\left\langle\overline{\Psi_{a}}|P| \Psi_{h}\right\rangle\left\langle\overline{\Psi_{h}}|Q| \Psi_{a}\right\rangle}{E+\left(\varepsilon_{a}-\varepsilon_{h}\right)+i \eta}\right],
$$

which shows that the unperturbed polarization includes not only particle-hole pairs but also pairs formed from the Dirac and Fermi states $\bar{\alpha} h$, when one substitutes $\tilde{G}_{H}$ in Eq.(7). The terms with $\bar{\alpha} h$ pairs would correspond to the Pauli-violating terms which appear if one works with $G_{H}$ in Eq.(7) [11]. There is no Pauli violation in the present approach because we work with the modified Green's function $\tilde{G}_{H}$.

In the time-dependent RMF (TDRMF) approach the no-sea approximation is imposed at each time. In the small amplitude limit the density $\rho(t)$ can be expanded as the density at $t=0$ and a small density change $\delta \rho(t)$ which can be calculated in a complete set of states given by the static solution of RMF. This complete set includes the positive as well as negative energy states. Thus, $\delta \rho$ has non-zero matrix elements $\delta \rho_{p h}$ and $\delta \rho_{\bar{\alpha} h}$. Therefore, the particle-hole space of RRPA must be built with both positive and negative energy states. The equivalence between the RRPA including the Dirac states and the small amplitude limit of the TDRMF with no-sea approximation can be formally shown [3].

In Eq.(8), one finds that the particle-hole energies between the occupied positive energy states and the Dirac states are usually two orders of magnitude larger than those involving only positive energy states. Furthermore, the matrix elements of $P$ and $Q$ between positive and negative energy states should be small because the positive (negative) energy states have a small lower (upper) component in their Dirac spinors. It is thus found that the $\bar{\alpha} h$ pairs make no visible contribution to the imaginary or to the real part of the unperturbed polarization in the energy region of the nuclear collective excitations. This is the reason why the Dirac states were usually not included in most previous works of the RRPA. However, we will see in the next sections that the correlated polarization operator can be affected by the Dirac states when the particle-hole interaction depends on the $\sigma$ meson, i.e., in the case of isoscalar excitations.

\section{CONSTRAINED RMF}

In the non-relativistic approach there is a complete equivalence between the static polarizability calculated in RPA and that deduced from a constrained Hartree-Fock calculation, and this property can be used as a check of consistency of the calculations. The same property will be used here to compare the static polarizabilities of RRPA and constrained RMF and to investigate the importance of the inclusion of Dirac states in the RRPA. The constrained RMF method was used in Ref. [12] to evaluate the ISGMR energies within the Walecka's linear model. In this section we discuss the constrained RMF results obtained with effective lagrangians having different types of self-interactions in the $\sigma$ and $\omega$ fields.

In general, a constrained RMF equation with an external field $\lambda Q$ is described as:

$$
(H(\lambda)+\lambda Q)|\lambda\rangle=E(\lambda)|\lambda\rangle
$$

where $H(\lambda)$ is the RMF hamiltonian, which depends on the parameter $\lambda$ due to the selfconsistency of the single-particle states, $|\lambda\rangle$ and $E(\lambda)$ are the ground state and corresponding energy of $H(\lambda)+\lambda Q$, respectively. At $\lambda=0$ the first order variation of $\langle\lambda|Q| \lambda\rangle$ with respect 
to $\lambda$, i.e., the static polarizability is equal to the second order variation of $E(\lambda)$. The same static polarizability can be calculated as twice the inverse energy-weighted sum rule $m_{-1}$ of RRPA,

$$
\begin{aligned}
\left.\frac{\partial\langle\lambda|Q| \lambda\rangle}{\partial \lambda}\right|_{\lambda=0} & =\left.\frac{\partial^{2} E_{\lambda}}{\partial \lambda^{2}}\right|_{\lambda=0} \\
& =2 \sum_{n \neq 0} \frac{\left\langle 0|Q| \Psi_{n}\right\rangle\left\langle\Psi_{n}|Q| 0\right\rangle}{E_{n}-E_{0}}
\end{aligned}
$$

The second line of Eq.(10) shows that the polarizability can be deduced from the real part of $\Pi$ calculated at $E=0$. In the case of the ISGMR, the operator $Q$ is simply $\gamma_{0} r^{2}$ and the RRPA $m_{-1}$ sum rule can also be obtained by performing a spherically constrained RMF calculation. Note that, if one replaces in Eq.(9) the self-consistent hamiltonian $H(\lambda)$ by the fixed hamiltonian $H_{0}$ of the unconstrained RMF solution, then Eq.(10) gives the $m_{-1}$ sum rule of the unperturbed response corresponding to the polarization operator $\Pi_{0}$.

The effective Lagrangians we use here include the scalar meson $\sigma$, vector meson $\omega$, isovector vector meson $\rho$ and the photon field $A^{\mu}$. Self-interactions in the $\sigma$ and $\omega$ fields are introduced in order to simulate a density dependence and hence to lower the compression modulus of nuclear matter [13]:

$$
\begin{aligned}
\mathcal{L}= & \bar{\Psi}\left(i \gamma^{\mu} \partial_{\mu}-M_{N}-g_{\sigma} \sigma-g_{\omega} \gamma^{\mu} \omega_{\mu}-g_{\rho} \tau^{a} \gamma^{\mu} \rho_{\mu}^{a}-e \gamma^{\mu} A_{\mu} \frac{1}{2}\left(1-\tau_{3}\right)\right) \Psi \\
& +\frac{1}{2} \partial^{\mu} \sigma \partial_{\mu} \sigma-U_{\sigma}-\frac{1}{4} W^{\mu \nu} W_{\mu \nu}+U_{\omega}+\frac{1}{2} m_{\rho}^{2} \rho^{a \mu} \rho_{\mu}^{a}-\frac{1}{4} R^{a \mu \nu} R_{\mu \nu}^{a}-\frac{1}{4} F^{\mu \nu} F_{\mu \nu}
\end{aligned}
$$

where

$$
\begin{aligned}
W^{\mu \nu} & =\partial^{\mu} \omega^{\nu}-\partial^{\nu} \omega^{\mu}, \\
R^{a \mu \nu} & =\partial^{\mu} \rho^{a \nu}-\partial^{\mu} \rho^{a \nu}+g_{\rho} \epsilon^{a b c} \rho^{b \mu} \rho^{c \nu}, \\
F^{\mu \nu} & =\partial^{\mu} A^{\nu}-\partial^{\nu} A^{\mu}, \\
U_{\sigma}=\frac{1}{2} m_{\sigma}^{2} \sigma^{2}+\frac{1}{3} g_{2} \sigma^{3} & +\frac{1}{4} g_{3} \sigma^{4}, \quad U_{\omega}=\frac{1}{2} m_{\omega}^{2} \omega^{\mu} \omega_{\mu}+\frac{1}{4} c_{3}\left(\omega^{\mu} \omega_{\mu}\right)^{2} .
\end{aligned}
$$

We briefly outline the procedure of the numerical calculations. The nucleon mean fields are calculated by solving the Dirac-Hartree equation in coordinate space self-consistently in the RMF approximation. All single-particle states (unoccupied and occupied) are calculated by diagonalizing the self-consistent mean fields in a harmonic oscillator basis with a proper choice of the oscillator length $b$ and the number of shells $(N=12)$. An averaging parameter $\Delta=2 \mathrm{MeV}$ is introduced as the imaginary part of the energy to smooth out the response functions. Calculations are performed with different non-linear lagrangians which span a wide range of values of the nuclear incompressibility: NL1 [14], NLSH [15], NL3 [16] and TM1 [17]. They generally give good descriptions of the ground state properties. The linear Walecka model HS [18] is also considered for comparison because of its high incompressibility. In the constrained RMF calculations we use a small step $\Delta \lambda=0.5 \times 10^{-4} \mathrm{fm}^{-3}$ in order to calculate the derivatives with sufficient accuracy. 
The values of the inverse energy-weighted sum rules calculated in constrained RMF and in RRPA are compared in Table 1 for the case of ISGMR in ${ }^{208} \mathrm{~Pb}$. We find a good agreement(within 1\%) between the first two columns, which is a numerical check of the accuracy of the constrained RMF calculations. The values of $m_{-1}$ in the third column are calculated by integrating the inverse energy-weighted ISGMR strength (from 0 to $60 \mathrm{MeV}$ ). The last column corresponds to one half of the real part of the response function at zero energy. The agreement between the last two columns simply indicates that the dispersion relation between the real and imaginary parts of $\Pi$ or $\Pi_{0}$ is well obeyed numerically. The comparison between the constrained RMF and the RRPA provides a severe check for the RRPA method used in this paper. It is found that the agreement is quite good (within 1\%) for the unperturbed cases while the difference becomes a few percent for the RRPA cases. We find that the discrepancies between RRPA and constrained RMF are mainly due to the Coulomb force. If one switches off the Coulomb force the agreement becomes perfect (less than $1 \%$ in all cases). The expansion of the wave functions on the harmonic oscillator basis may not be accurate enough to account for the long range effects of the Coulomb force especially for the Dirac states with an attractive Coulomb interaction. A more sophisticated method would be to calculate the Green's functions directly in coordinate space [19].

The discrepancies between the RRPA and the constrained RMF are dramatic if one does not take the Dirac states into account as one can see in row III of Table 1. The large contribution to the RRPA strengths from the Dirac states can be understood in the following way. In the relativistic approach the nucleon potential is obtained by a strong cancellation between the isoscalar scalar and vector potentials, and so do the particle-hole residual interactions in the isoscalar channel. Due to the $\gamma$-matrix structure the matrix elements of the residual interaction between particle-hole pairs through the exchange of scalar and vector mesons are largely cancelled to two orders of magnitude. However, this is not true for pairs built with Fermi and Dirac states because the large component of the wave function is the upper component (lower component) for a Fermi (Dirac) state. The orthogonality between positive and negative energy states with the same quantum numbers is mainly due to the cancellation between the upper and lower components. Again, due to the $\gamma$-matrix structure the matrix elements between Fermi and Dirac states through the exchange of vector mesons $(\omega$ or $\rho$ ) are small whereas those corresponding to a scalar meson are large. These scalar meson matrix elements are repulsive and they can overcome the large energy gap between Dirac and Fermi states. 


\section{FIGURES}

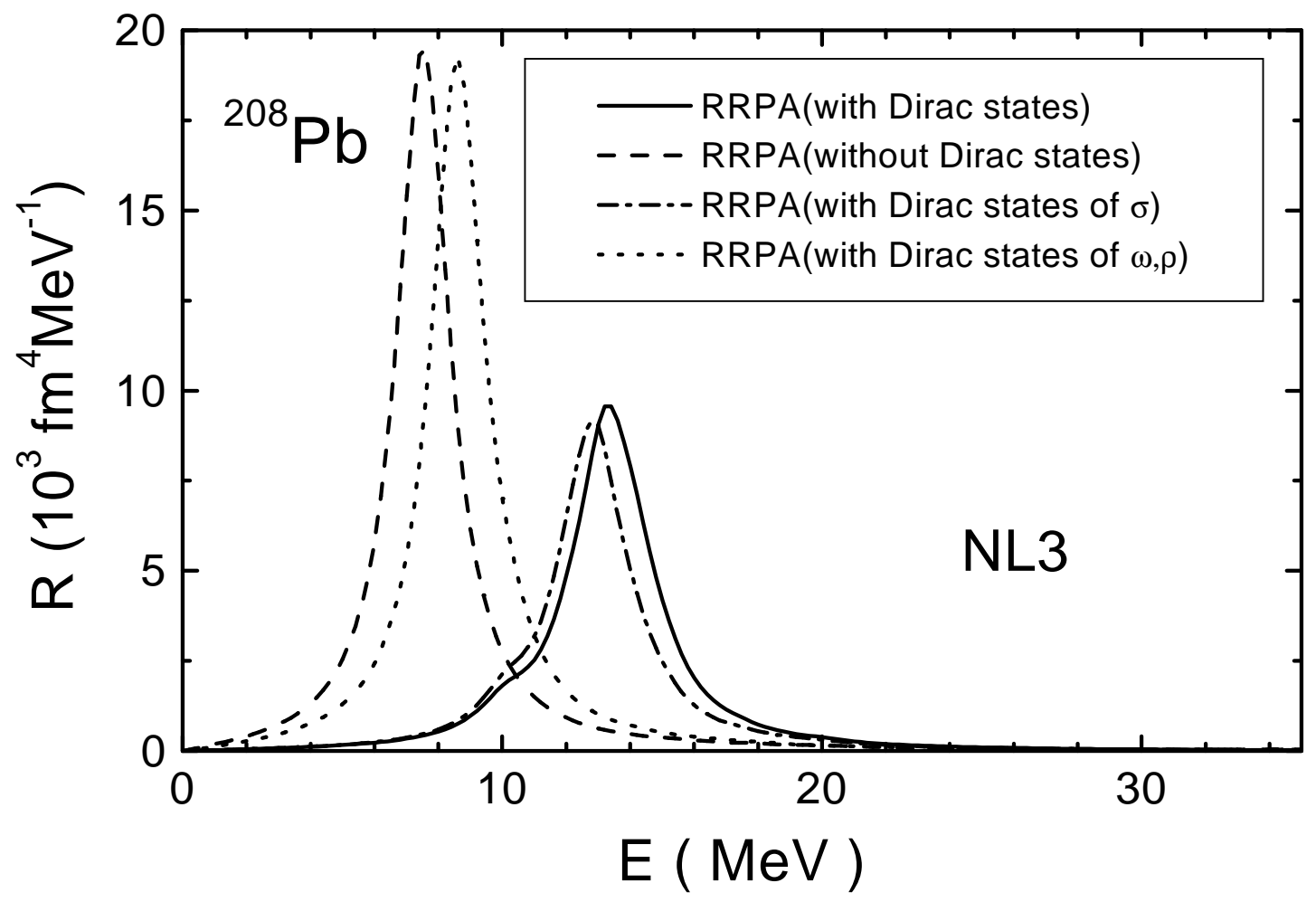

FIG. 1. ISGMR strength distributions in ${ }^{208} \mathrm{~Pb}$ calculated with NL3 parametrization. The solid and long-dashed curves are the RRPA strengths with and without Dirac states, respectively. The short-dashed (dash-dotted) curve corresponds to calculations where only vector mesons (scalar mesons) are included in the couplings between Fermi and Dirac states.

The large effects of Dirac states are illustrated in Fig.1. We separate the contributions from scalar and vector mesons for the $\bar{\alpha} h$ pairs and we plot the monopole strengths in ${ }^{208} \mathrm{~Pb}$, taking NL3 as an example. The solid and long-dashed curves are the RRPA strengths with and without Dirac states, respectively. They are markedly separated. The ISGMR strength is pushed strongly down without the Dirac state contributions. The short-dashed curve is the RRPA strength with the contributions of $\bar{\alpha} h$ pairs coming from the vector meson only. It is more or less the same as the case without Dirac states. The dash-dotted curve is calculated with only the scalar meson contributions to the $\bar{\alpha} h$ pairs. It is clearly seen that the contributions of the scalar meson in the $\bar{\alpha} h$ pairs dominate whereas those due to the vector meson are negligible.

It can be concluded that the inclusion of only the positive particle-hole pairs in the isoscalar modes, where the isoscalar mesons play an important role, would provide a too strong attraction and therefore, a too low isoscalar giant resonance energy. This will reflect in a too large polarizability as shown in Table 1. On the other hand, the contributions from Dirac states can be neglected in the case of isovector modes where the isovector-vector meson dominates. 


\section{MONOPOLE COMPRESSION MODE}

The value of the compression modulus $K_{\infty}$ of nuclear matter is one of the most important issues of nuclear physics. The generally accepted procedure for determining $K_{\infty}$ is to calculate isoscalar monopole energies, as well as other properties of finite nuclei using various microscopic models in order to select those which can describe correctly the data and to use these models for constructing the nuclear matter equation of state [20]. Here, we examine the predictions of the various effective lagrangians in the framework of RRPA. In this work we adopt the mean field approach and therefore the effects of $2 \mathrm{p}-2 \mathrm{~h}$ couplings [21] are not included.
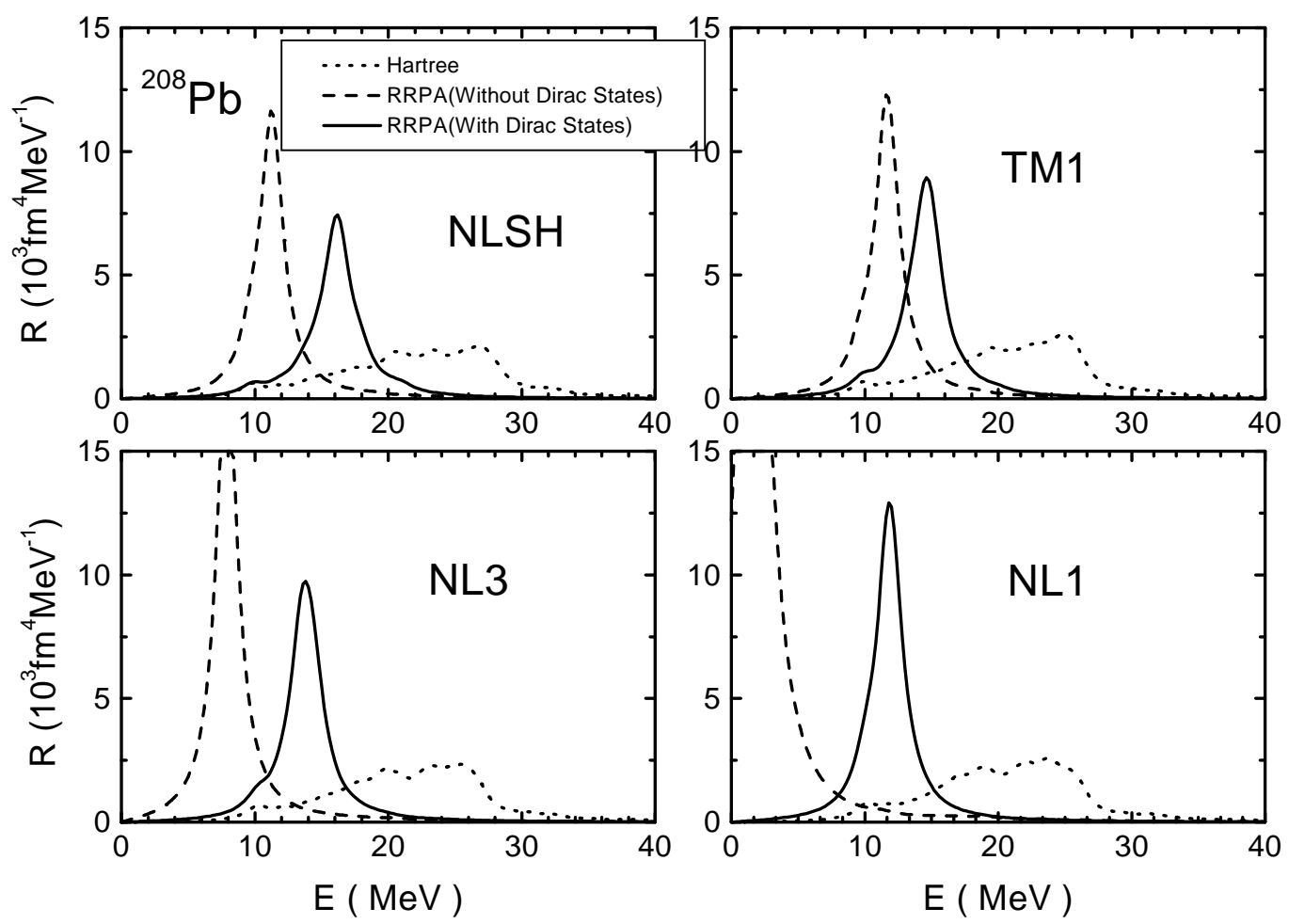

FIG. 2. ISGMR strength distributions in ${ }^{208} \mathrm{~Pb}$ calculated with different parameter sets.

We are interested in establishing a link between the measured energies of compression modes in nuclei and $K_{\infty}$. The most favorable situation is met in heavy nuclei where the giant monopole resonance is less broad than in medium and light nuclei. In the latter case, the Landau fragmentation is quite strong due to the weakness of the particle-hole interaction in the isoscalar monopole channel. In order to have a comparison with non-relativistic RPA results and with TDRMF results we concentrate on ${ }^{208} \mathrm{~Pb}$ and ${ }^{144} \mathrm{Sm}$ where the experimental giant monopole energies are $14.2 \pm 0.3$ and $15.4 \pm 0.3 \mathrm{MeV}$, respectively [22]. The calculations are performed with 5 effective Lagrangians whose compression modulus span a wide range of values: $\operatorname{NL} 1\left(K_{\infty}=211 \mathrm{MeV}\right)$, NL3 $\left(K_{\infty}=272 \mathrm{MeV}\right)$, TM1 $\left(K_{\infty}=281 \mathrm{MeV}\right)$, NLSH $\left(K_{\infty}=355 \mathrm{MeV}\right)$, HS $\left(K_{\infty}=545 \mathrm{MeV}\right)$. The inclusion of negative energy states in the 
RRPA calculations necessarily involves the choice of a cut-off energy in the Dirac sea. The bottom of the potential on the negative side is around $-150 \mathrm{MeV}$, typically. We have checked that the results are stable if we move the cut-off between $-300 \mathrm{MeV}$ and $-900 \mathrm{MeV}$.

The RRPA monopole strengths with and without Dirac states are shown in Fig.2 for different effective Lagrangians. The strengths are shifted down strongly to the lower energies when only the positive energy particle-hole pairs are included. In some parametrization, such as NL1, the strong attractive residual interaction produced by the positive particlehole pairs would bring the system beyond a critical point, where the system is not stable. In the complete RRPA calculations with the Dirac states included the strengths are shifted to higher energies and the unstabilities disappear. In a recent work [23] such an effect due to the Dirac states has been shown analytically in the framework of nuclear matter and the Walecka's linear model.

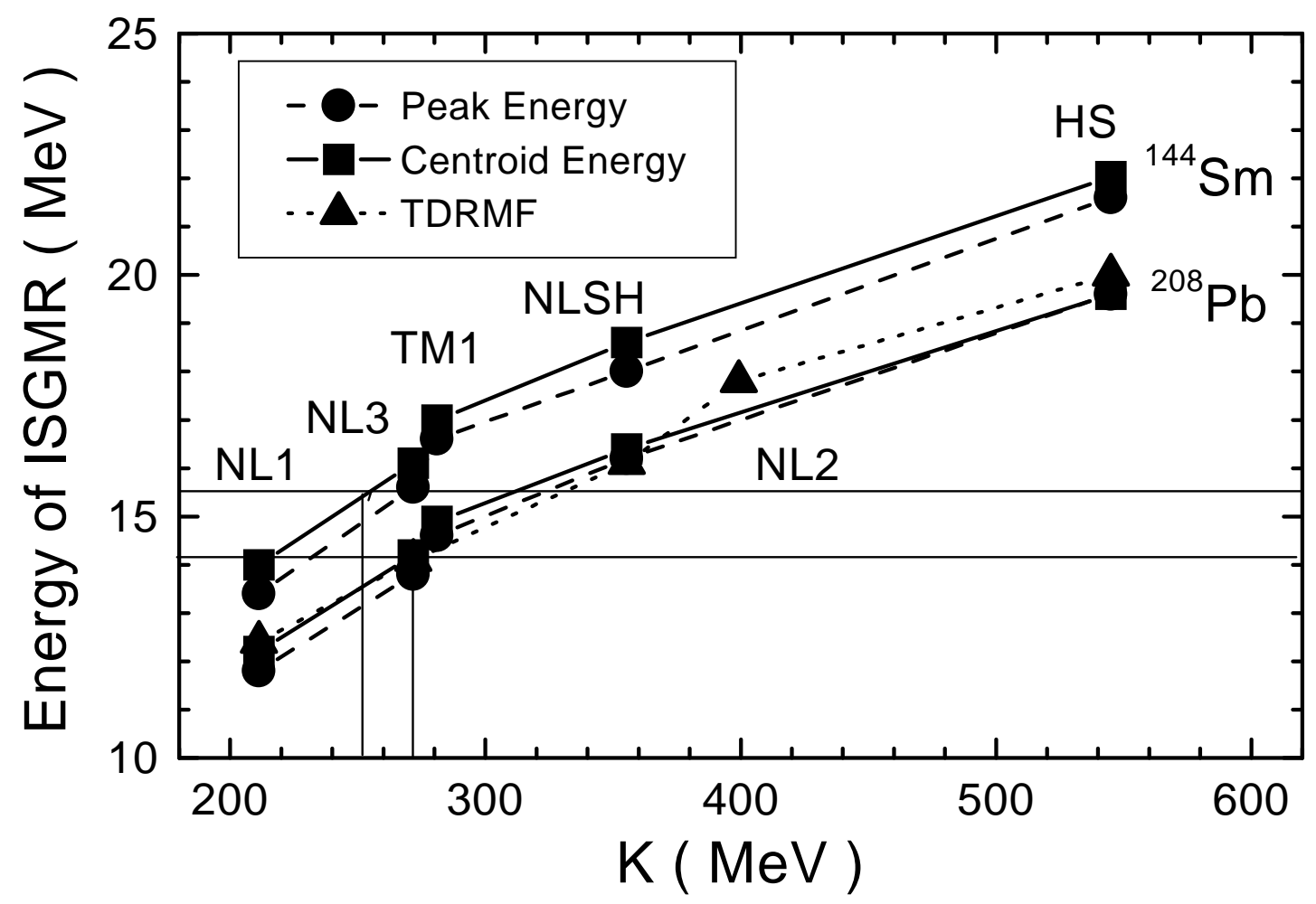

FIG. 3. RRPA peaks and centroid energies of the ISGMR in ${ }^{208} \mathrm{~Pb}$ and ${ }^{144} \mathrm{Sm}$ as functions of $K_{\infty}$. TDRMF results for ${ }^{208} \mathrm{~Pb}[24]$ are also shown. The experimental values for ${ }^{208} \mathrm{~Pb}$ and ${ }^{144} \mathrm{Sm}$ are $\mathrm{E}=14.2 \pm 0.3$ and $15.4 \pm 0.3 \mathrm{MeV}$, respectively[22].

In Fig. 3 we show the RRPA monopole energies in ${ }^{208} \mathrm{~Pb}$ and ${ }^{144} \mathrm{Sm}$ as functions of $K_{\infty}$. In each case, the difference between peak and centroid energies is generally small due to the fact that the strength is mostly concentrated in the giant resonance region. This difference would be larger in lighter nuclei. The ISGMR energies calculated in TDRMF [24] are also plotted in figure 3 in comparison with our RRPA results. Note that the present results differ from those of Ref. [25] where the space-like contributions of vector mesons were not 
included in the particle-hole residual interaction. A good agreement of the ISGMR energies from the two approaches is obtained. The dependence on $K_{\infty}$ is nearly linear, similarly to what was obtained in non-relativistic RPA calculations performed with Skyrme and Gogny interactions [26]. From Fig.3 it can be concluded that a value of $K_{\infty}$ close to $250 \sim 270$ $\mathrm{MeV}$ would bring the RRPA results in good agreement with experiment.

\section{DIPOLE COMPRESSION MODE}

The isoscalar giant dipole compression mode, or squeezing mode is mainly built on $3 \hbar \omega$ excitations. It was already observed a long time ago [27] and it has been measured recently with increased accuracy by $\left(\alpha, \alpha^{\prime}\right)$ scattering [28] in several medium and heavy nuclei. We

have performed RRPA calculations of this mode in ${ }^{208} \mathrm{~Pb}$. An external field [29] $\gamma_{0}\left(r^{3}-\eta r\right) Y_{10}$ with $\eta=\frac{5}{3}<r^{2}>$ is used as a probe. The second term in the operator is introduced to remove a large amount of spurious components coming from the admixture of the center-ofmass state. The value of $\eta$ is determined by a condition of translational invariance.

It is found that there is a large amount of strength in the $1 \hbar \omega$ region around $10 \mathrm{MeV}$. The main peak (in terms of energy-weighted strength) is at about $26 \mathrm{MeV}$ for the case of NL3. This peak moves up with increasing incompressibility. This general pattern of results, namely a sizable fraction of strength at lower energies and a giant resonance peak well above $20 \mathrm{MeV}$ is also found in non-relativistic RPA calculations with Skyrme interactions [29,21]. This is at variance with the experimental findings [28] where no strength is observed in the $10-15 \mathrm{MeV}$ region and where the ISGDR peak is close to $20 \mathrm{MeV}$ in ${ }^{208} \mathrm{~Pb}$. It should be noted, however, that in the continuum-RPA calculation of ref. [30] the low-lying strength could be eliminated by a particular subtraction procedure, but the ISGDR peak still remains above $20 \mathrm{MeV}$.

In Fig. 4 are shown the calculated peak energies as a function of the compression modulus. The centroid energies calculated in the giant resonance region (20-40 MeV) are also displayed. The peak and centroid energies are generally quite close except for the NLSH case where the giant resonance is much Landau- fragmented. The calculated energies have a weaker $K_{\infty}$-dependence than in the monopole case. The measured value [28] of centroid energy is 20.3 MeV. One can see that a model like NL3 which is doing rather well for the ISGMR energy is now off by $5 \mathrm{MeV}$ in the ISGDR case. This disagreement between experiment and models for the ISGDR case is the next puzzle to be solved. 


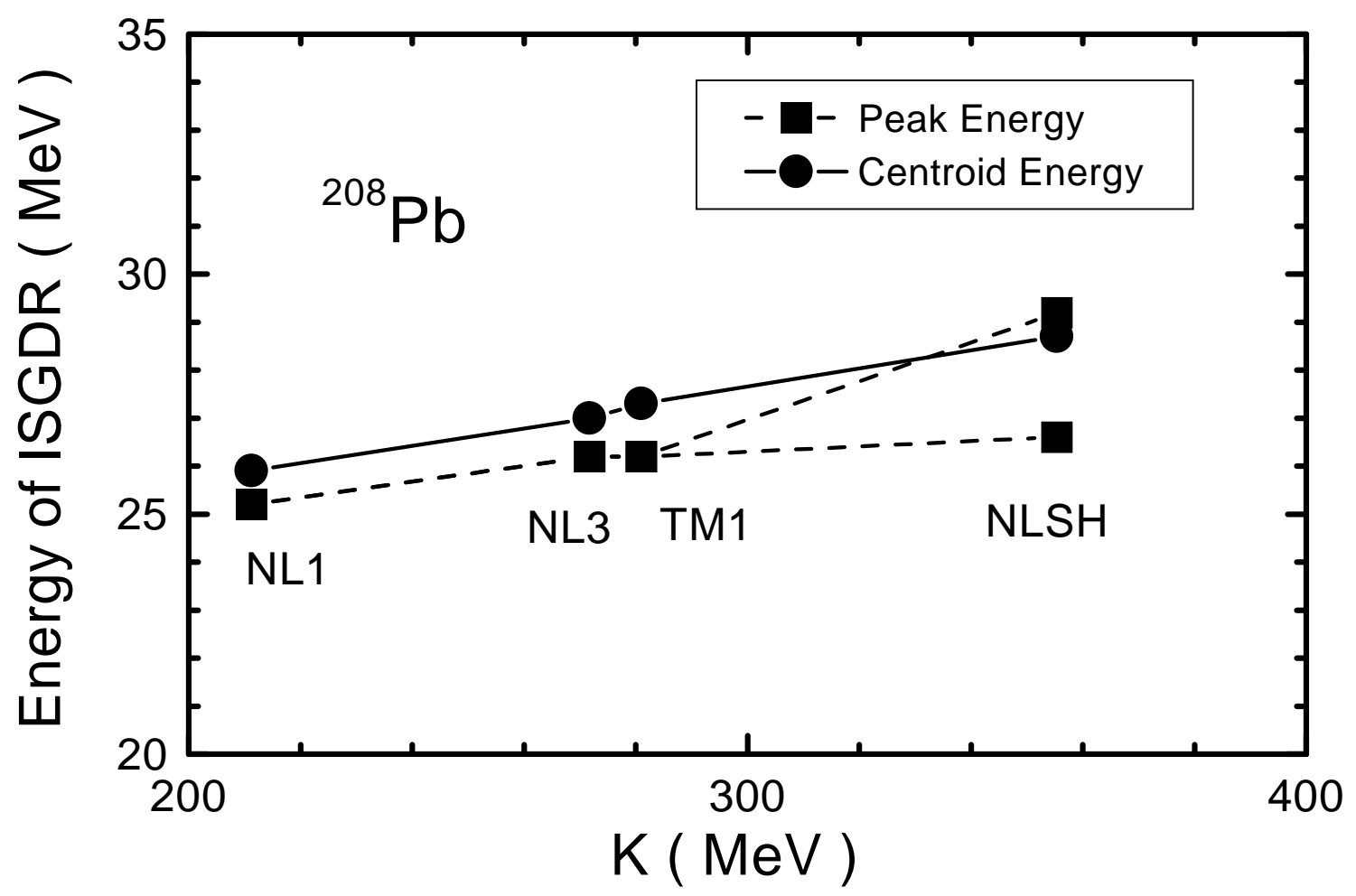

FIG. 4. Peak and centroid energies (in the $20-40 \mathrm{MeV}$ region) of the ISGDR in ${ }^{208} \mathrm{~Pb}$ as a function of $K_{\infty}$. The experimental centroid energy is $\mathrm{E}=20.3 \mathrm{MeV}$.

\section{SUMMARY AND CONCLUSION}

In this paper we have studied an RRPA approach built on the RMF in the framework of the relativistic baryon-meson field theory. The effective Lagrangians with non-linear meson self-interactions, which describe well the ground state properties of finite nuclei as well as the nuclear matter matter properties are adopted. The calculation is fully consistent in the sense that the single-particle basis and the particle-hole couplings are obtained from the same effective Lagrangian. In a no-sea approximation, a consistent treatment of the RRPA must include both the particle-hole pairs built with positive energy states and the $\bar{\alpha} h$ pairs formed with the occupied Fermi sea and the empty Dirac sea states.

The importance of the inclusion of the Dirac sea states is examined in detail in the case of the monopole compression mode. We have used the constrained RMF method with a $r^{2}$ constraint as a check of RRPA calculations. With the inclusion of Dirac sea states the RRPA static polarizability, or the inverse energy-weighted sum rule, is in agreement with the constrained RMF result whereas the RRPA polarizability becomes much larger (it can even be negative for some effective Lagrangians) if the Dirac states are omitted. In general, the Dirac sea states must play an important role in the isoscalar modes. The reason is that the repulsive contributions of the $\bar{\alpha} h$ pairs through exchange of the isoscalar-scalar meson dominate. Their effect is to shift the isoscalar giant resonances to higher energies. 
The remaining small discrepancies between RRPA and constrained RMF polarizabilities when the Coulomb field is turned on seem to indicate that the harmonic oscillator basis used here may not be fully appropriate. A proper treatment of the Coulomb interaction in Dirac sea states for heavy nuclei is required to get accurate results because of the long range attraction for the negative energy states. Employing the Green's function technique in coordinate space and carrying out a continuum RRPA [19] will probably improve the accuracy of results. A good agreement of ISGMR energies calculated in RRPA and TDRMF is obtained when we include the Dirac state contributions in the calculation of RRPA.

The comparison of the isoscalar monopole data for heavy nuclei, namely ${ }^{208} \mathrm{~Pb}$ and ${ }^{144} \mathrm{Sm}$, with calculations performed with different parameter sets indicates that the compression modulus of nuclear matter is in the range of $250 \sim 270 \mathrm{MeV}$. These values are somewhat larger than those predicted by non-relativistic RPA models [26].

The main open problem is now to understand the ISGDR energies. The discrepancies between experiment and predictions for this mode are the largest ever found for isoscalar giant resonances described in non-relativistic RPA or RRPA. It is unlikely that the coupling to $2 \mathrm{p}-2 \mathrm{~h}$ configurations [21] can account for them.

Acknowledgments We would like to thank U. Garg and T. Suzuki for discussions. Z.Y.M. acknowledges the support of the SPM Department of CNRS and the hospitality of IPNOrsay. The hospitality of RIKEN during completion of the work is acknowledged by Z.Y.M. and N.V.G. This work is partially supported by the China National Natural Science Foundation under Grant No. 19675070, 19847002, and by the Bundesministerium für Bildung und Forschung under contract No. 06TM875. D.V. thanks the Deutsche Forschungsgemeinschaft for funding a guest professorship at the TUM. 


\section{REFERENCES}

[1] G.F.Bertsch and S.F.Tsai, Phys. Reports 18, 125 (1975).

[2] P.Ring and P.Schuck, The Nuclear Many-body Problem (Springer, Heidelberg,1980).

[3] P. Ring et al., to be published.

[4] R.Brockmann and R.Machleidt, Phys. Rev.C 42, 1965 (1990).

[5] Z.Y.Ma, N.Van Giai and H.Toki, Phys. Rev.C 55, 2385 (1997).

[6] Z.Y.Ma, H.Toki and N.Van Giai, Nucl. Phys.A 627, 1 (1997).

[7] S.A.Chin, Ann. Physics (N.Y.)108, 301 (1977).

[8] J.F.Dawson and R.J.Furnstahl, Phys. Rev.C 42, 2009 (1990).

[9] N. Van Giai, Z.Y. Ma, H. Toki and B.Q. Chen, Nucl. Phys. A 649, 37c (1999).

[10] D. Vretenar, P. Ring, G.A. Lalazissis and N. Paar, Nucl. Phys. A 649, 29c (1999).

[11] M.L'Huillier and N. Van Giai, Phys. Rev.C 39, 2022 (1989).

[12] T.Maruyama and T.Suzuki, Phys. Lett.B219, 43 (1989).

[13] J. Boguta and A.R. Bodmer, Nucl. Phys. A 292, 413 (1977).

[14] P.G.Rheinhard, M.Rufa, J.Maruhn, W.Greiner and J.Friedrich, Z. Phys.A323, 13 (1986).

[15] M.M.Sharma, M.A.Nagarajan and P.Ring, Phys. Lett.B312, 377 (1993).

[16] G.A.Lalazissis, J.König and P.Ring, Phys. Rev.C 55, 540 (1997).

[17] Y.Sugahara and H.Toki, Nucl. Phys.A 579, 557 (1994).

[18] C.J.Horowitz and B.D.Serot, Nucl. Phys.A 368, 503 (1981).

[19] K.Wehrberger and F.Beck, Phys. Rev. C 37, 1148 (1988).

[20] J.P.Blaizot, Phys. Reports 64, 171 (1980).

[21] G.Colò, N.Van Giai, P.F.Bortignon and M.R.Quaglia, The RIKEN Review23, p.39, Eds. N.Dinh Dang and S.Yamaji (Riken, Tokyo, 1999).

[22] D.H.Youngblood, H.L.Clark and Y.W.Lui, Phys. Rev. Lett.82, 691 (1999).

[23] H. Kurasawa and T. Suzuki, Phys. Lett.B474, 262 (2000).

[24] D. Vretenar, G. A. Lalazissis, R. Behnsch, W. Pöschl and P. Ring, Nucl. Phys. A621, 853 (1997).

[25] N.Van Giai, Z. Y. Ma, The RIKEN Review23, p.69, Eds. N.Dinh Dang and S.Yamaji (Riken, Tokyo, 1999).

[26] J.P.Blaizot, J.F.Berger, J.Dechargé and M.Girod, Nucl. Phys.A 591, 435 (1995).

[27] H.P.Morsch et al., Phys. Rev. Lett.45, 337 (1980); C.Djalali et al., Nucl. Phys.A 380, $42(1982)$.

[28] U.Garg, Nucl. Phys.A 649, 66c (1999); H.L.Clark et al., Nucl. Phys.A 649, 57c (1999);

U.Garg, private communication.

[29] N.Van Giai and H.Sagawa, Nucl. Phys.A 371, 1 (1981).

[30] I.Hamamoto, H.Sagawa and X.Z.Zhang, Phys. Rev.C 57, R1064 (1998). 


\section{TABLES}

TABLE I. The ISGMR inverse energy-weighted sum rule in ${ }^{208} \mathrm{~Pb}$. The rows with I,II,III refer to the results of unperturbed (Hartree), RRPA with Dirac states and without Dirac states, respectively. All values are in $10^{3} \mathrm{fm}^{4} \mathrm{MeV}^{-1}$.

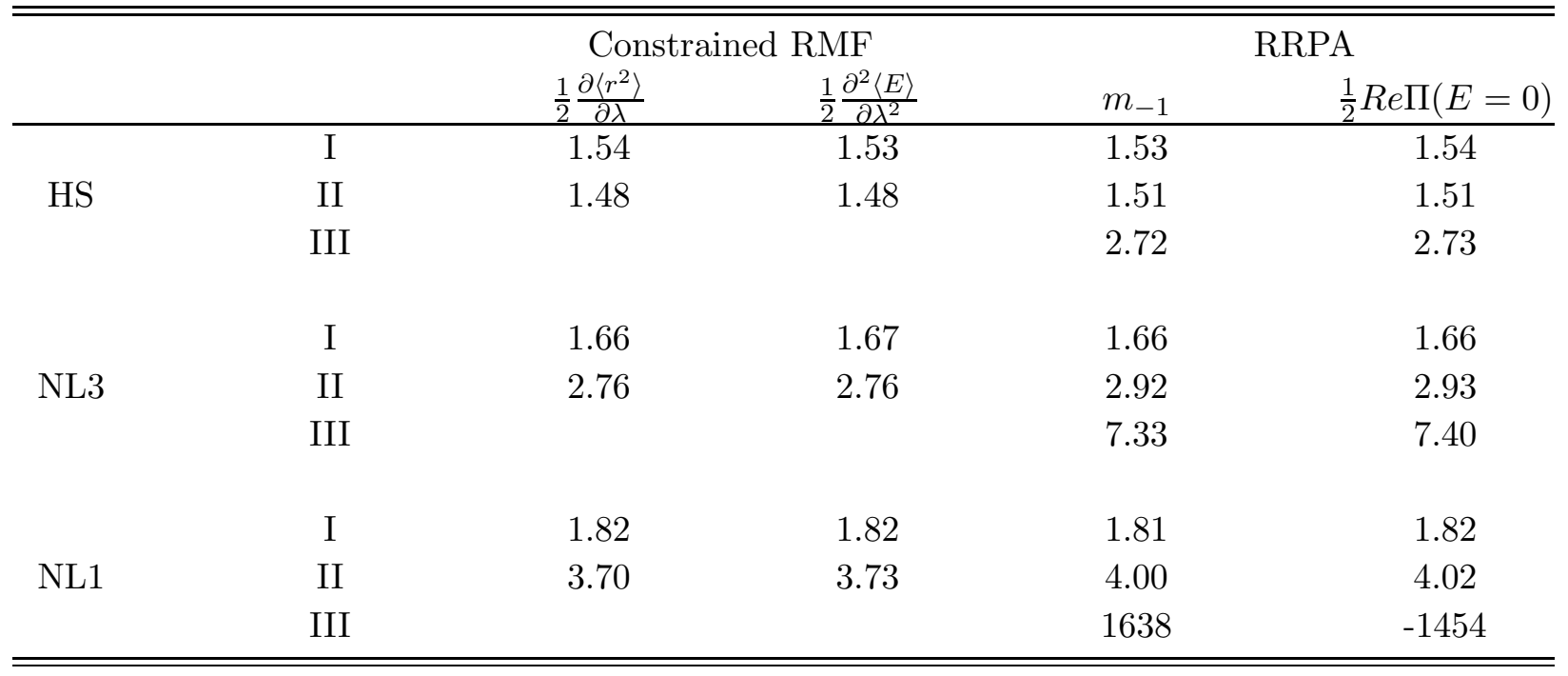

